\title{
Quadcopter thrust optimization with ducted-propeller
}

\author{
Endrowednes Kuantama ${ }^{1 *}$, Radu Tarca $^{2}$ \\ ${ }^{1}$ Engineering Doctoral School University of Oradea-Romania, endrowednes@gmail.com \\ ${ }^{2}$ Mechatronics Department University of Oradea-Romania, rtarca@uoradea.ro
}

\begin{abstract}
In relation to quadcopter body frame model, propeller can be categorized into propeller with ducted and without ducted. This study present differences between those two using CFD (Computational Fluid Dynamics) method. Both categories utilize two blade-propeller with diameter of $406(\mathrm{~mm})$. Propeller rotation generates acceleration per time unit on the volume of air. Based on the behavior of generated air velocity, ducted propeller can be modeled into three versions. The generated thrust and performance on each model were calculated to determine the best model. The use of ducted propeller increases the total weight of quadcopter and also total thrust. The influence of this modeling were analyzed in detail with variation of angular velocity propeller from 1000 (rpm) to 9000 (rpm). Besides the distance between propeller tip and ducted barrier, the size of ducted is also an important part in thrust optimization and total weight minimization of quadcopter.
\end{abstract}

\section{Introduction}

Quadcopter aerodynamic movement is based on thrust generated by four propellers. Movement control can be established by varying the angular velocity of each propeller independently to achieve desired attitude and altitude H. Guang, G. Hoffmann, S. Waslander, and C.J.Tomlin [1]. The magnitude of thrust depends on propeller size, magnitude of pitch, and angle of attack. At this point, analyses were done to improve quadcopter performance R. Czyba and G. Szafranski [2], V. Hrishikeshavan and I. Chopra [3]. This study focused on the design perspective of quadcopter frame by using ducted and non-ducted propeller. By using ductedpropeller, disturbance effect may be decreased and thrust can be increased and optimized, although the total weight of quadcopter will be increased J. Pereira, D. Bawek, S. Westfall, and I. Chopra [4], V. Hrishikeshavan, J. Sirohi, M. Tishchenko, and I. Chopra [5]. The most important part of ducted-propeller is how to create model and minimizing added weight of barrier for propeller while optimizing propeller performance. The distance slot between duct and propeller's tip is important part to minimize the drag effect and eliminate the friction point of barrier.

The analysis was done to observe quadcopter performance in terms of aerodynamic movement and disturbance occurred on quadcopter based on thrust generated by rotational speed. By using CFD (Computational Fluid Dynamics), both air pressure and air velocity generated by propeller rotation can be analyzed and calculated. Air motion of propeller in relation to the distance between propellers on symmetrical frame of quadcopter was analyzed with variation on rotational speed from 1000-9000 (rpm). Commercial propeller with diameter of $406(\mathrm{~mm})$ was chosen as model and analyzed in this system.

\section{Quadcopter propeller model and algorithm}

A two-blade propeller was designed using CAD (Computer-aided Design) and the model was analyzed using CFD method to observe air trajectory generated by propeller rotation. The magnitude of lift force from propeller depends on angular velocity and angle of attack, which in this design is $3^{\circ}$. The acceleration of air generated from propeller rotation can produce thrust. According to airfoil theory, it is known that pressure over the top of propeller is lower than the pressure below the propeller Y. Yang, L. L. M. Veldhuis, and G. Eitelberg [6]. Fig. 1. shows the propulsion of propeller and flow stream of the propeller. The distribution of air velocity along the propeller's blade tip through the vortex center shows that the air velocity on the tip is lower than the hub because the surface area propeller in hub is larger than in the tip. The downstream of the air

\footnotetext{
*Corresponding author: endrowednes@gmail.com
} 
on surface area propeller will lead downward until the pressure eventually return to free stream conditions. In fact, not all air trajectory lead down but they are sucked back by propeller, which occurs on the tip located in $\mathrm{x}$ and $y= \pm 200$ (mm). By knowing the air flow trajectory, one can do modeling, analyze the ducted propeller and compare each other.
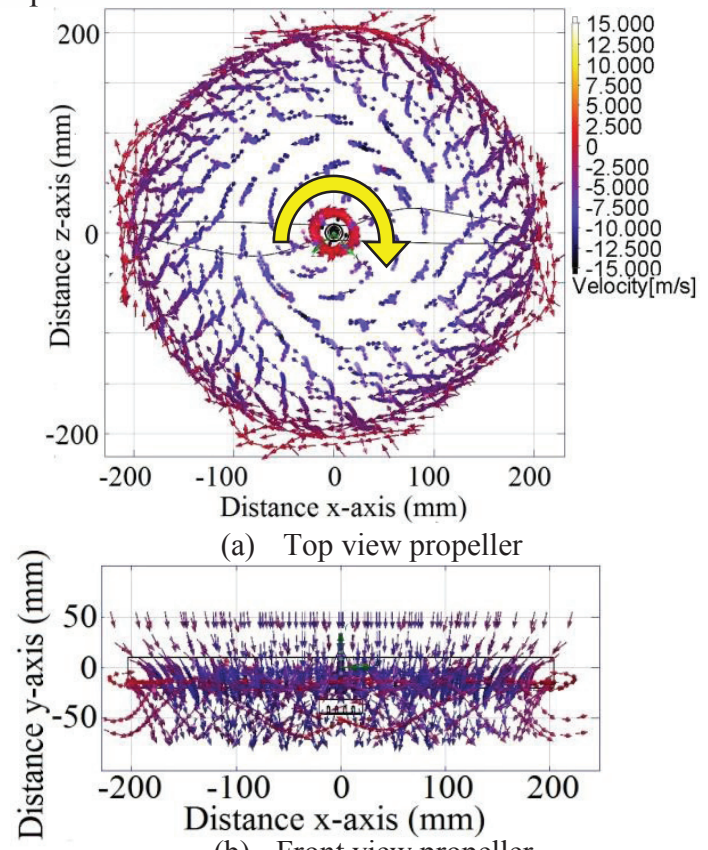

(b) Front view propeller

Fig. 1. Propeller air trajectory

Lift force on propeller, also known as thrust, can be calculated using momentum theory as seen on Equation (1). The generated thrust depends on propeller's air velocity which moves from upstream to downstream, and the air velocity itself depends on rotor's angular velocity and the change in momentum. There are two types of velocity generated from propeller's rotation, i.e. axial induced velocity $\left(v_{i}\right)$ and free stream velocity $\left(v_{\infty}\right)$ . Free stream velocity comes from air turbulence on wind which affect propeller H. A. Kutty and P. Rajendran [7]. Air density of $1.225\left(\mathrm{~kg} \cdot \mathrm{m}^{-3}\right)$ represented by symbol $\rho$ and surface area propeller represented by symbol A .

$$
T=\rho A\left[\left(2 v_{i}+v_{\infty}\right)-v_{\infty}\right]
$$

For ducted lift propeller, thrust can be calculated by adding variable duct diffusion ratio $(\sigma)$ as seen on Equation (2) and (3). Non-ducted propeller has diffusion ratio of 0.5 and ducted propeller has diffusion ratio between 0.5-1 Y. Jiang, B. Zhang, and T. Huang [8]. Air velocity on ducted propeller can be calculated based on velocity generated by propeller $\left(v_{\mathrm{p}}\right)$ and velocity in downstream propeller $\left(v_{\mathrm{d}}\right)$.

$$
\begin{gathered}
\mathbf{T}=\mathbf{T}_{\mathbf{p}}+\mathbf{T}_{\mathbf{d}}=\rho A v_{\mathbf{p}} v_{d} \\
v_{p}=\sigma v_{d}
\end{gathered}
$$

From flow trajectory or forward velocity $\left(\mathrm{V}_{\mathrm{T}}\right)$ generated by rotation of rotor propeller (n), vortex impact and performance of the propeller can be analyzed through parameter advance ratio $(\mathrm{J})$ and coefficient thrust $\left(\mathrm{T}_{\mathrm{C}}\right)$, as shown in Equation (4) and (5). These equations are the standard formulas for the pressure generated by propeller rotation with diameter (D) corresponding to propeller rotation area S. B. Heinzen, C. E. Hall, and A. Gopalarthnam [9], Y. Yang, D. Ragni, L Veldhuis, and G. Eitelberge [10].

$$
\mathbf{J}=\frac{\mathbf{V}_{\mathbf{T}}}{\mathbf{n D}}
$$

These algorithms $\mathbf{T}_{\mathbf{c}}=\frac{\mathbf{T}}{\mathbf{\rho n}^{\mathbf{2}} \mathbf{D}^{\mathbf{4}}}$ will be used to differentiate between quadcopter performance using ducted and non-ducted propeller. Through CFD, air velocity generated by propeller can be measured, thus the magnitude of generated thrust can be analyzed.

\section{Ducted propeller design and analysis}

Based on air trajectory as the result of non-ducted propeller rotation, one can design three types of ducted model, presented in Fig. 2. The main focus of the modeling is minimum material addition for ducted to make as little weight gain as possible, thus optimal thrust result can be achieved. The space between tip propeller and ducted is $10(\mathrm{~mm})$ in each model.

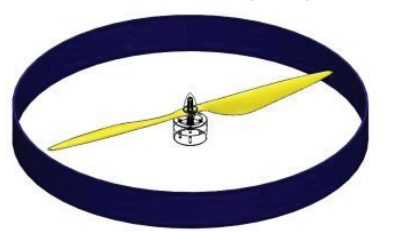

(a) Type $\alpha$

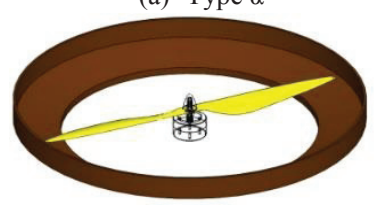

(c) Type $\gamma$

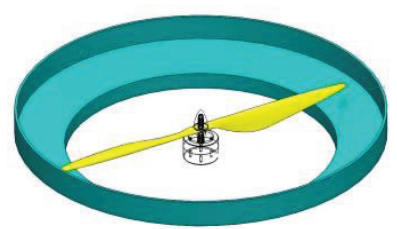

(b) Type $\beta$

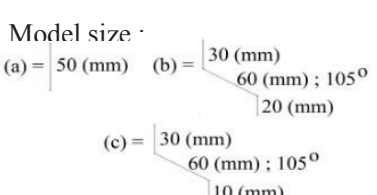
$10(\mathrm{~mm})$

Fig. 2. Ducted propeller model

The modeling was designed based on airflow trajectory as the result of propeller rotation as seen in Fig.1. Surface area propeller around hub located on $(-140 \leq \mathrm{x} \leq 140)$ and $(-140 \leq \mathrm{z} \leq 140)$ generated the biggest air velocity and moving straight down to produce thrust. Meanwhile, on the propeller tip area, not all generated air velocity moving downward but they are being sucked back by propeller with circular air movement which occur on position $(-3 \leq \mathrm{y} \leq 3)$. Ducted propeller type $\alpha$ and type $\beta$ has little difference in

\footnotetext{
Corresponding author: endrowednes@gmail.com
} 
the bottom which was done to lessen the material load while still optimizing generated thrust. From three models of ducted propeller, the generated air velocity will be analyzed to calculate thrust value of each model. Surface area propeller in this model is $0.317\left(\mathrm{~m}^{2}\right)$.

\section{Quadcopter thrust and air trajectory analysis}

CFD was used to analyze the induced velocity generated by propeller with variation between 1000 (rpm) and 9000 (rpm) with scale of 1000 (rpm). In this simulation, wind effect or outside disturbance was not taken into account thus free stream velocity was dispensed $\left(v_{\infty}=0\right)$. The higher the propeller rotation, the bigger the induced velocity will be. However in this study, the generated air velocity will be compared with normal propeller, ducted and non-ducted. Ducted propeller itself was designed with three different models. Fig. 3. shows the induced velocities generated by each model.

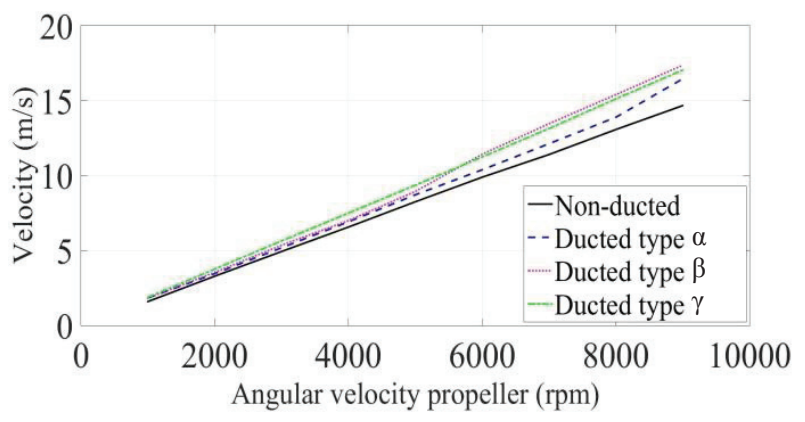

Fig. 3. Induced velocities generated by propeller

With the same propeller size and low angular velocity $(\leq 4000(\mathrm{rpm}))$, induced velocities generated between ducted and non-ducted propeller are not differ significantly. However for angular velocity higher than $4000(\mathrm{rpm})$, differences in induced velocities are clearly seen. Ducted propeller type $\beta$ and type $\gamma$ has differences in terms of surface area ducted in which type $\beta$ surface area is $0.276\left(\mathrm{~m}^{2}\right)$ and type $\gamma$ surface area is $0.255\left(\mathrm{~m}^{2}\right)$. Both ducted types produce nearly identical (not significantly differ) induced vorticities, because type $\gamma$ was designed to reduce the weight of the cover material used.

In this study, the type of material used was not taken into account but from the surface area ducted it can be seen that the $\alpha$ type is lighter than the other ducted type of modeling. From induced velocities generated by propeller, the magnitude of thrust generated by propeller rotation can be calculated using Equation (1) and the result can be seen in Fig. 4. This equation is enough to calculate the generated thrust. Besides that, by using CFD, velocity in downstream propeller can also be analyzed where the magnitude must be smaller than the induced velocities generated by propeller.

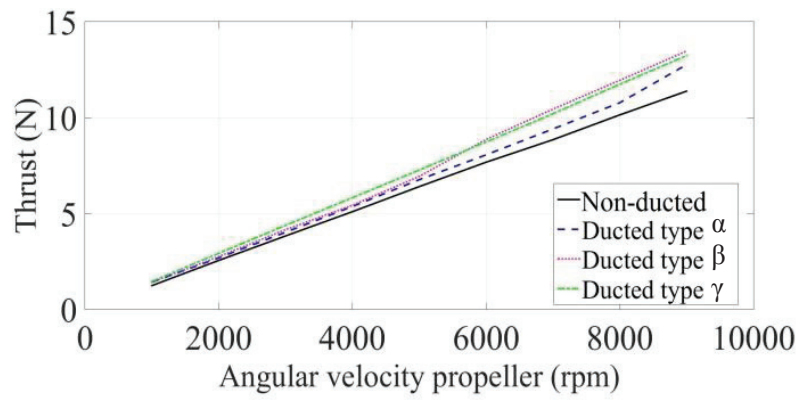

Fig. 4. Thrust propeller

Thrust optimization can be done by adding cover on propeller. However not all ducted propeller models can work to improve the thrust performance. From the test result, the ducted angle must be greater than $90^{\circ}$ to ensure that the resulting air velocity leads downward. This modeling uses an angle of $105^{\circ}$ to improve the propeller performance. From the thrust calculation, it can be seen that thrust optimization on ducted propeller can occur on high rotation propeller $(\geq 4000(\mathrm{rpm}))$ with an increase in thrust value of $0.27-1.38(\mathrm{~N})$ for propeller type $\alpha$ and $0.73-2.1(\mathrm{~N})$ for propeller type $\beta$ and $\gamma$. From previous research, angular velocity propeller 5000 (rpm) was suitable with folded size body frame with size of $560(\mathrm{~mm})$ in square size E. Kuantama, D. Craciun, and R. Tarca [11]. With the same angular velocity, ducted propeller was analyzed to examine its effect on quadcopter as a whole. With ducted propeller, the size of body frame or distance between propeller may be smaller compared with non-ducted. Body frame with width and length size 460 (mm) x 460 (mm) was designed as seen on Fig. 5. and Fig. 6.

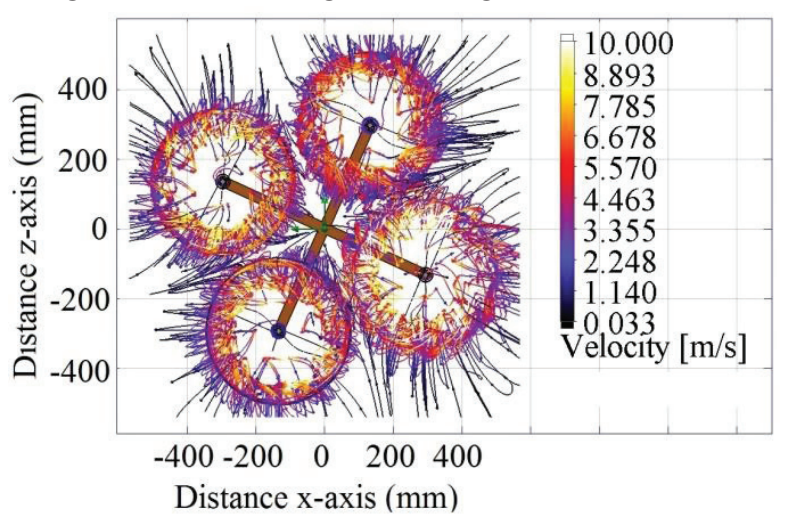

Fig. 5. Quadcopter without propeller cover (Non-ducted)

By observing the air flow trajectory generated by each propeller, it can be seen that without ducted, air velocity generated by each propeller are affecting each other $\left(v_{\infty} \neq 0\right)$ thus resulting in inevitable instability of quadcopter. Whereas on ducted propeller, air flow trajectory directly moves downward which means the body frame size of $460(\mathrm{~mm}) \times 460(\mathrm{~mm})$ (width $\mathrm{x}$ length) can be used for quadcopter with maximum angular velocity of 5000 (rpm).

\footnotetext{
Corresponding author: endrowednes@gmail.com
} 


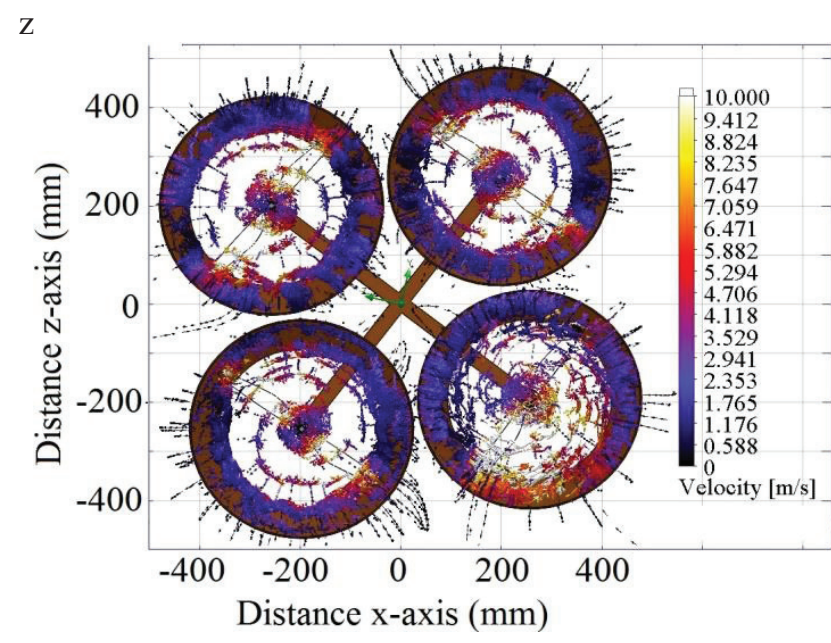

Fig. 6. Quadcopter with ducted type $\gamma$

Overall the efficiency of quadcopter propeller model can be calculated based on advance ratio propeller, thrust coefficient propeller and rotor power. The greater the advance ratio value and thrust coefficient, the greater the propeller efficiency. However, the greater the power needed to rotate the propeller, the smaller the total efficiency of propeller or vice versa O. Gur [12]. Using Equation (4) and (5), non-ducted propeller produce thrust coefficient of 0.031 with advance ratio of 0.24 and ducted propeller type $\gamma$ generate thrust coefficient of 0.037 with advance ratio of 0.28 . If the power generated between both models has the same value, then the ducted propeller type $\gamma$ has better efficiency value. Deeper analysis can be done by calculating the correlated power rotor with the resulting thrust which is not the focus of this study.

\section{Conclusion}

Propeller with size of 406 ( $\mathrm{mm}$ ) can be optimized by adding cover on propeller or also known as ducted propeller. With maximum angular velocity propeller of 9000 (rpm), ducted propeller type $\alpha$ generate thrust of $1.38(\mathrm{~N})$ and propeller type $\beta$ and $\gamma$ generate thrust of 2.1 $(\mathrm{N})$. This means that the addition of ducted frame material should not be more than 140 gram-force (gf) for each propeller cover type $\alpha$, wheras in type $\gamma$ the addition of ducted frame material should not exceed 214 (gf). Type $\gamma$ is better than type $\beta$ because it has lighter mass while produce the same thrust. In terms of quadcopter body frame size, ducted propeller can be designed smaller than the non-ducted propeller. Body frame size was based on maximum angular velocity of propeller rotation thus the air velocity produced between propeller will not interfere with each other. For angular velocity 5000 (rpm), a quadcopter ducted body frame with size of $460(\mathrm{~mm})$ in square size can be used.

Assuming the power required to produce propeller rotation with the same angular velocity, ducted propeller has a better efficiency than the non-ducted because the resulting airflow trajectory moves downward to generate maximum thrust with minimum disturbance.

\section{Acknowledgment}

This work has been funded under the LEADERS Erasmus Mundus Grant (agreement number 20140855/001-001) by European Commission, through the Education, Audio-visual and Culture Executive Agency, in the Action Plan 2 for the years 2014-2018, and supported by PNCDI III Programme P2 - Transfer of knowledge to the economic operator (Bridge Grant PNIII-P2 2.1 BG-2016-0296) funded by UEFISCDI, Romania.

\section{References}

[1] H. Guang, G. Hoffmann, S. Waslander, and C.J.Tomlin, "Aerodynamics and Control of Autonomous Quadcopters in Aggressive Maneuvering," International Conference on Robotics and Automation, 2009, pp. 3277-3282.

[2] R. Czyba, and G. Szafranski, "Control Structure Impact on the Flying Performance of the Multi-Rotor VTOL Platform - Design, Analysis and Experimental Validation," International Journal of Advanced Robotic Systems , 2012, pp. 1-9.

[3] V. Hrishikeshavan, and I. Chopra, "Performance, Flight Testing of Shrounded Rotor Micro Air Vehicle in Edgewise Gusts," Journal of Aircraft, vol. 49, 2012, pp. 193-205.

[4] J. Pereira, D. Bawek, S. Westfall, and I. Chopra, "Design and Development of a Quad-Shrouded-Rotor Micro Air Vehicle," American Helicopter Society $65^{\text {th }}$ Annual Forum, 2009.

[5] V. Hrishikeshavan, J. Sirohi, M. Tishchenko, and I. Chopra, "Design and Development of a Shrouded Rotor Micro Air Vehicle with Anti-torque Vanes," Journal of the American Helicopter Society, vol. 56, 2011, pp. 1-11.

[6] Y. Yang, L. L. M. Veldhuis, and G. Eitelberg, " Investigation of Propeller Induced Ground Vortices by Numerical and Experimental Methods." AIAA Applied Aerodynamics Conference, 2015, pp. 1-12.

[7] H. A. Kutty and P. Rajendran, "3D CFD Simulation and Experimental Validation of Small APC Slow Flyer Propeller Blade," Aerospace Journal, vol. 4, pp. 1-11, 2017.

[8] Y. Jiang, B. Zhang, and T. Huang, "CFD Study of an AnnularDucted Fan Lift System for VTOL Aircraft," Aerospace Journal, vol. 2, 2015, pp. 555-580.

[9] S. B. Heinzen, C. E. Hall, and A. Gopalarthnam, "Development and Testing of a Passive Variable-Pitch Propeller," Journal of Aircraft, vol. 52, 2015, pp. 748-763.

[10] Y. Yang, D. Ragni, L Veldhuis, and G. Eitelberge, "Propeller Induced Ground Vortex," 28 $8^{\text {th }}$ International Congress of The Aeronautical Sciences, 2012, pp. 1-11.

[11] E. Kuantama, D. Craciun, and R. Tarca, "Quadcopter Body Frame Model and Analysis," Annals of The University of Oradea, 2016, pp. 71-74.

[12] O. Gur, "Maximum Propeller Efficiency Estimation," Journal of Aircraft, vol. 51, 2015, pp. 2035-2038 